\title{
Role of Diffusion Tensor Imaging In Intractable Unilateral Mesial Temporal Lobe Epilepsy
}

\author{
Amarnath $\mathrm{C}^{1}$, Bhoopathy $\mathrm{R} \mathrm{M}^{2}$, Suhasini $\mathrm{B}^{1}$, Prem Chand ${ }^{1}$, Gopinathan $\mathrm{K}^{3}$, \\ Philson $\mathbf{J}^{4}$, Kanimozhi D ${ }^{1}$ \\ ${ }^{I}$ (Stanley medical college, Chennai, India) \\ 2 (Tamil Nadu Government Multi Super speciality Hospital, Chennai, India) \\ ${ }^{3}$ (Kilpauk medical college, Chennai, India) \\ ${ }^{4}$ (Scans World research \& education institute, Chennai, India)
}

\begin{abstract}
We evaluated 21 patients with unilateral MTLS by using DTI, MRI, clinical and EEG parameters to assess changes in DTI metrics in hippocampal formation, white matter tracts and gray matter nuclei. Out of these 10 were Right sided and 11 were left sided MTLS. We compared the mean diffusivity (trace D) and fractional anisotropy (FA) from symmetrical voxels by sampling the following areas namely hippocampus, parahippocampal white matter, fimbriae and fornix middle cerebellar peduncle, corpus callosum, uncinate fasciculus, inferior fronto occipital fasciculus, superior longitudinal fasciculus, , anterior and posterior cingulum, thalamus, internal capsule, caudate and lentiform nucleus. There was statistically significant decrease in FA values with increased mean diffusivity was seen in ipsilateral hippocampus, parahippocampal white matter, fimbrae and fornix, inferior fronto- occipital fiber and posterior cingulate. On comparison with controls, we noted increased MD and decreased FA values in contralateral hippocampus, parahippocampal white matter, fimbriae and fornix middle cerebellar peduncle, corpus callosum, uncinate fasciculus, inferior fronto occipital fasciculus, superior longitudinal fasciculus, and cingulum. DTI is highly sensitive to micro structural changes in brain that underlie epilepsy. It shows microstructural abnormalities beyond the involved hippocampus extending to ipsilateral and contralateral major white matter tracts which is not apparent on conventional MRI.
\end{abstract}

Keywords: DTI, Mesial Temporal lobe Epilepsy, Mean diffusivity, Fractional Anisotropy, Hippocampal sclerosis.

Abbreviations: DTI -Diffusion Tensor Imaging, MTLS -Unilateral mesial temporal lobe sclerosis, FA Fractional anisotropy, MD-Mean diffusivity.

\section{Introduction}

A seizure is defined as the signs/symptoms caused by the abnormal excessive neuronal activity in the brain. Epilepsy is the tendency to have unprovoked seizures. About $1 \%$ of people worldwide are suffering from epilepsy1 and the sensitivity of conventional MRI with the current epilepsy protocol in identifying the epileptogenic focus is only slightly greater than $50 \% 2$. So there is a need for additional sequences like diffusion tensor imaging in cryptogenic cases of epilepsy.

The most common form of focal epilepsy is Temporal lobe epilepsy. The etiology can be varied like hippocampal sclerosis, malformations of cortical development, mass lesions, AV malformations, gliosis etc. Previous studies with diffusion tensor imaging have shown increased apparent diffusion coefficient and decreased fractional anisotropy in the seizure focus2. Though the origin of seizure activity is focal, there is widespread propagation of synchronized neuronal firing in seizure disorders via neuronal networks and other cortical and subcortical regions of the brain are affected3. These widespread changes may be reflected as altered diffusion tensor imaging metrics. Diffusion tensor imaging non invasively assess the microstructure of brain tissue, By introducing directionality into diffusion weighted images, diffusion tensor images are obtained1

Mean diffusivity (MD) and Fractional Anisotropy ( FA) are the two parameters derived from it. MD reflects the average magnitude of molecular displacement by diffusion and Fractional Anisotropy ( FA) reflects the directionality of molecular displacement. Pathological conditions of brain alters MD and Fractional Anisotropy ( FA) values. In this study Diffusion Tensor Imaging( DTI) metrics are combined with statistical parameter mapping to explore the wide spread white matter networks and grey matters to gain further insight into the organization and structural abnormalities associated with temporal lobe epilepsy. Confounding factors like age of onset, duration of seizure, frequency of seizure and duration of treatment are taken into consideration. 
We compared the Fractional Anisotropy (FA) and mean diffusivity (MD) of ipsilateral and contralateral hippocampus, other white matter tracts as well as deep grey matter of the patients with intractable unilateral temporal lobe epilepsy.

\section{Subjects And Methods}

The study was approved by local ethical committee and informed written consent was taken from all patients and healthy volunteers. The study was conducted on 21 patients (10 males and 11 females) aged between 17 to 49 years a mean of 33.48 years, with clinical features of temporal lobe epilepsy refractory to medical treatment referred to our department for MRI examination. MRI examination confirmed mesial temporal sclerosis in all 21 patients, all patients underwent interictal EEG examination. Patients were included in the study irrespective of their previous treatment status. Patients with dual intraaxial structural abnormalities other than temporal lobe and, those with major psychiatric disorders were excluded from study. 10 controls (5 males and 5 female) undergoing MRI examination for indication other than epilepsy were taken for baseline Diffusion Tensor Imaging (DTI) values.

\subsection{Imaging protocol}

The examination was performed in 1.5T SIEMENS (Germany), MAGNETOM AERA MRI system using standard head coil. Conventional imaging protocol consist of T1W sagittal, T2W axial and coronal and FLAIR coronal. Parameters for T1W image were TR: 429ms, TE: $12 \mathrm{~ms}$, Matrix $320 * 256$, slice thickness of 4 mm with no interslice gap. T2W images TR: $3000 \mathrm{~ms}$, TE $80 \mathrm{~ms}$, and Matrix 420*270 slice thickness of $4 \mathrm{~mm}$ with no interslice gap. FLAIR imaging was done with TR: $11000 \mathrm{ms,} \mathrm{TE:} 100 \mathrm{~ms}$ TI: 2500, Matrix 244*156 slice thickness of $4 \mathrm{~mm}$ with no interslice gap. Diffusi on weighted Echo planar images were acquired in the same system with following parameters: TR: $3500 \mathrm{~ms}$, TE $83 \mathrm{~ms}$, matrix 256 X 258 slice thickness of 3 $\mathrm{mm}$ with no interslice gap, FOV 224 and B- value 800. Diffusion sensitive gradients were applied in 21 optimized collinear direction. T1W 3D MIRAGE sequence imaging was done with TR: $8.4 \mathrm{~ms}$, TE: $4.1 \mathrm{~ms}$, Matrix $256 * 256$. Raw data were corrected for geometric distortion secondary to eddy current. Maps of Fractional Anisotropy (FA) and mean diffusivity (MD) were generated from diffusion weighted images and superimposed on 3D SPGR sequence.

\subsection{Imaging analysis}

All DTI images were transferred to a workstation where image reconstruction and post processing analysis was performed. ROIs of similar size were placed in color coded FA map superimposed over isotropic T1W images over bilateral hippocampi, body of fornix, para hippocampal white matter, anterior limb, genu and posterior limb of internal capsule, external capsule, genu, body and splenium of corpus callosum, thalamus, lentiform nucleus and head of caudate nucleus, frontal and occipital regions of superior longitudinal fasciculus, temporal and occipital regions of inferior longitudinal fasciculus, uncinate fasciculus, middle cerebellar peduncle, arcuate fasciculus and cingulum.

FA and ADC values from each of these ROI was calculated. The ROIs were placed on the axial images at the level of foramen of Munro for anterior limb, genu and posterior limb of internal capsule, thalamus, lentiform nucleus and head of caudate nucleus. For corpus callosum, frontal and occipital regions of superior longitudinal fasciculus (Fig .1), ROIs were placed on the axial images when the region was maximally seen.

For hippocampus, para hippocampal white matter, fornix and uncinate fasciculus coronal images were used. Parasagittal images were used to locate the inferior longitudinal fasciculus.

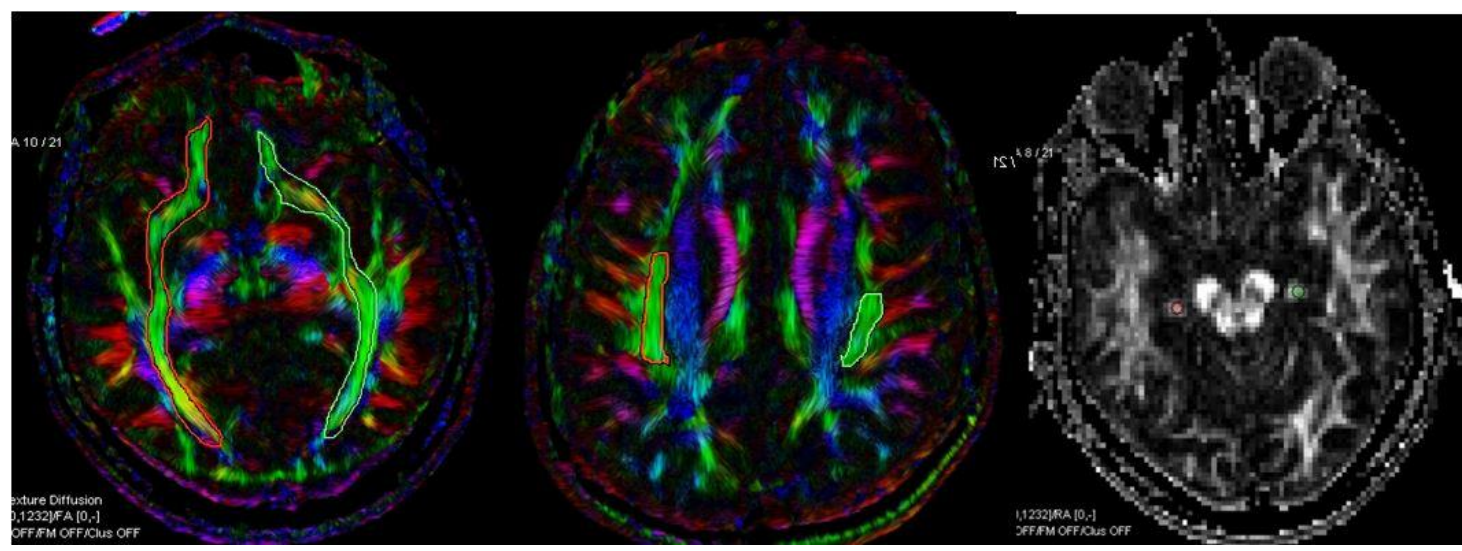

Fig 1. Showing color coded maps with Region of Interests (ROI) in superior longitudinal fasciculus and parahippocampal gyrus as decribed in the paragraph above 


\subsection{Data analysis}

Statistical analysis was performed with all patients as single group. For this analysis, images were normalized such that all lesions were left side (the images of patients with right HS were mirrored to the left side. We compared (1) difference of Fractional Anisotropy (FA) and MD values between right and left side of controls (2) the differences between ipsilateral and contralateral sides in patients (3) differences between ipsilateral with mean of the controls (4) differences between contralateral and mean of the controls. Variables were normally distributed and expressed as mean \pm SD. Comparison of normally distributed continuous variables between cases and controls was done by independent sample $\mathrm{T}$ test. Comparison of right and left sided variables within controls was done by paired $\mathrm{T}$ test. All the $\mathrm{P}$ values less than 0.05 were considered statistically significant. Data entry was done in MS excel worksheet. Data analysis was done by SPSS software version 23.0.

\section{Results}

The statistical analysis of mean diffusivity and fractional anisotropy of different regions of the brain shows there is statistical significant increase in Mean Diffusivity (MD) and decrease in Fractional Anisotropy (FA) in the ipsilateral Hippocampus, parahippocampal white matter, uncinate fasciculus, fimbrae and fornix. While significant increased mean diffusivity noted in inferior fronto-occipital fiber and significant decreased Fractional Anisotropy (FA) value noted in ipsilateral anterior limb of internal capsule as well anterior cingulate.

\begin{tabular}{|c|c|c|c|c|}
\hline & SITE & Number & $\begin{array}{l}\mathrm{MD} \text { value }(10-3 \\
\mathrm{mm} 2 / \mathrm{s})\end{array}$ & $\begin{array}{l}\text { Fractional Anisotropy } \\
\text { (FA) values }\end{array}$ \\
\hline \multirow[t]{3}{*}{ Hippocampus } & Ipsilateral & 21 & $0.93+/-0.079$ & $0.146+/-0.026$ \\
\hline & Contralateral & 21 & $0.83+/-.034$ & $0.161+/-0.027$ \\
\hline & $\mathrm{P}$ value ( Paired T test) & & 0.000 & 0.000 \\
\hline \multirow[t]{3}{*}{ Parahippocampus } & Ipsilateral & 21 & $0.84+/-0.039$ & $0.36+/-0.036$ \\
\hline & Contralateral & 21 & $0.81+/-0.038$ & $0.438+/-0.038$ \\
\hline & $\mathrm{P}$ value( Paired T test) & & 0.004 & 0.000 \\
\hline \multirow{3}{*}{$\begin{array}{l}\text { Uncinate } \\
\text { fasciculus }\end{array}$} & Ipsilateral & 21 & $0.90+/-0.085$ & $0.30+/-0.52$ \\
\hline & Contralateral & 21 & $0.81+/-0.073$ & $0.36+/-0.053$ \\
\hline & $\mathrm{P}$ value( Paired T test) & & 0.016 & 0.000 \\
\hline \multirow[t]{3}{*}{ Fimbrae and fornix } & Ipsilateral & 21 & $0.82+/-0.085$ & $0.36+/-0.064$ \\
\hline & Contralateral & 21 & $0.81+/-0.073$ & $0.37+/-0.05$ \\
\hline & $\mathrm{P}$ value & & 0.64 & 0.72 \\
\hline \multirow{3}{*}{$\begin{array}{ll}\text { Superior } & \text { longitudinal } \\
\text { fasciculus } & \end{array}$} & Ipsilateral & 21 & $0.85+/-0.05$ & $0.52+/-0.06$ \\
\hline & Contralateral & 21 & $0.81+/-0.57$ & $0.54+/-0.06$ \\
\hline & $\mathrm{P}$ value( Paired T test) & & 0.002 & 0.06 \\
\hline \multirow{3}{*}{$\begin{array}{ll}\text { Inferior } & \text { temporooccipital } \\
\text { fasciculus } & \end{array}$} & Ipsilateral & 21 & $0.83+/-0.046$ & $0.47+/-0.05$ \\
\hline & Contralateral & 21 & $0.81+/-0.05$ & $0.50+/-0.04$ \\
\hline & $\mathrm{P}$ value( Paired T test) & & 0.06 & 0.147 \\
\hline \multirow{3}{*}{$\begin{array}{l}\begin{array}{l}\text { Anterior limb of } \\
\text { capsule }\end{array} \\
\text { Internal }\end{array}$} & Ipsilateral & 21 & $0.74+/-0.05$ & $0.51+/-0.058$ \\
\hline & Contralateral & 21 & $0.74+/-0.057$ & $0.46+/-0.066$ \\
\hline & $\mathrm{P}$ value( Paired T test) & & 0.699 & 0.001 \\
\hline \multirow{3}{*}{$\begin{array}{l}\text { posterior limb of } \text { Internal } \\
\text { capsule }\end{array}$} & Ipsilateral & 21 & $0.72+/-0.028$ & $0.66+/-0.047$ \\
\hline & Contralateral & 21 & $0.71+/-0.041$ & $0.66+/-0.070$ \\
\hline & $\mathrm{P}$ value( Paired T test) & & 0.413 & 0.966 \\
\hline \multirow[t]{3}{*}{ Middle Cerebellar Peduncle } & Ipsilateral & 21 & $0.70+/-0.039$ & $0.70+/-0.07$ \\
\hline & Contralateral & 21 & $0.70+/-0.023$ & $0.73+/-0.08$ \\
\hline & $\mathrm{P}$ value( Paired T test) & & 0.806 & 0.296 \\
\hline \multirow[t]{3}{*}{ Anterior Cingulum } & Ipsilateral & 21 & $0.78+/-0.05$ & $0.34+/-0.07$ \\
\hline & Contralateral & 21 & $0.77+/-0.04$ & $0.38+/-0.07$ \\
\hline & $\mathrm{P}$ value( Paired T test) & & 0.487 & 0.001 \\
\hline \multirow[t]{3}{*}{ Posterior cingulum } & Ipsilateral & 21 & $0.79+/-0.068$ & $0.40+/-0.072$ \\
\hline & Contralateral & 21 & $0.76+/-0.06$ & $0.42+/-0.073$ \\
\hline & $\mathrm{P}$ value( Paired T test) & & 0.04 & 0.07 \\
\hline \multirow[t]{3}{*}{ Arcuate fasciculus } & Ipsilateral & 21 & $0.71+/-0.04$ & $0.51+/-0.08$ \\
\hline & Contralateral & 21 & $0.71+/-0.063$ & $0.54+/-0.09$ \\
\hline & $\mathrm{P}$ value( Paired T test) & & 0.867 & 0.106 \\
\hline \multirow[t]{3}{*}{ Thalamus } & Ipsilateral & 21 & $0.74+/-0.031$ & $0.25+/-0.026$ \\
\hline & Contralateral & 21 & $0.74+/-0.027$ & $0.27+/-0.026$ \\
\hline & $\mathrm{P}$ value( Paired T test) & & 0.92 & 0.03 \\
\hline \multirow[t]{3}{*}{ Caudate Nucleus } & Ipsilateral & 21 & $0.73+/-0.02$ & $0.16+/-0.05$ \\
\hline & Contralateral & 21 & $0.72+/-0.03$ & $0.17+/-0.051$ \\
\hline & $\mathrm{P}$ value( Paired T test) & & 0.216 & 0.12 \\
\hline \multirow[t]{3}{*}{ Lentiform Nucleus } & Ipsilateral & 21 & $0.73+/-0.04$ & $0.13+/-0.02$ \\
\hline & Contralateral & 21 & $0.73+/-0.05$ & $0.13+/-0.02$ \\
\hline & $\mathrm{P}$ value( Paired T test) & & 0.514 & 0.65 \\
\hline
\end{tabular}

Table 1 - Mean MD and FA values in patient group (mean $+/-\mathrm{SD})$ with paired test $\mathrm{P}$ Values. 
When the mean diffusivity and Fractional Anisotropy (FA) of patients are compared with healthy volunteers there was increased in mean diffusivity and decreased Fractional Anisotropy (FA) value contralateral side Hippocampus, parahippocampal white matter and uncinate fasciculus. Statistical analysis shows no significant changes in fimbrae and fornix, middle cerebral peduncle, posterior internal capsule, superior longitudinal fibers, anterior cingulate, thalamus, lentiform nucleus, and head of caudate.

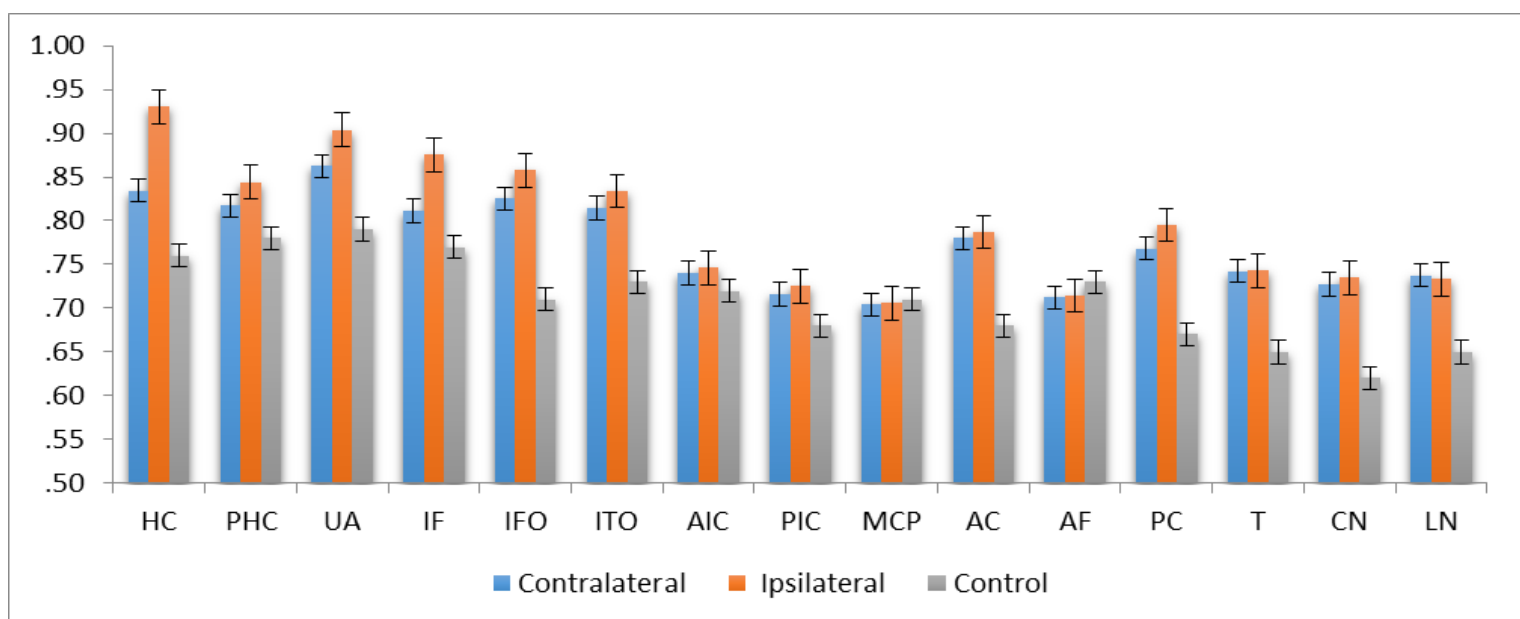

Fig .2 - Mean MD values with standard deviation for each patient and mean of controls. In patients higher MD values noted in ipsilateral hippocampus ( $\mathrm{HC}$ ), parahippocampal white matter (PHC) fornix (IF), uncinate fasciculus ( UF) anterior limb (AIC)and posterior limb(PIC) of internal capsule, superior longitudinal (IFO) and inferior Temporo-occipital (ITO fasciculus, middle cerebellar peduncle (MCP), anterior cingulum (AC)and posterior cingulum (PC), thalamus (T), caudate nucleus $(\mathrm{CN})$ and lentiform nucleus (LN). X- Axis mean diffusivity (MD) value (10-3 mm2/s). As compare to mean of controls MD values are increased in ipsilateral as well as contralateral side of each region though changes are more towards the ipsilateral side.

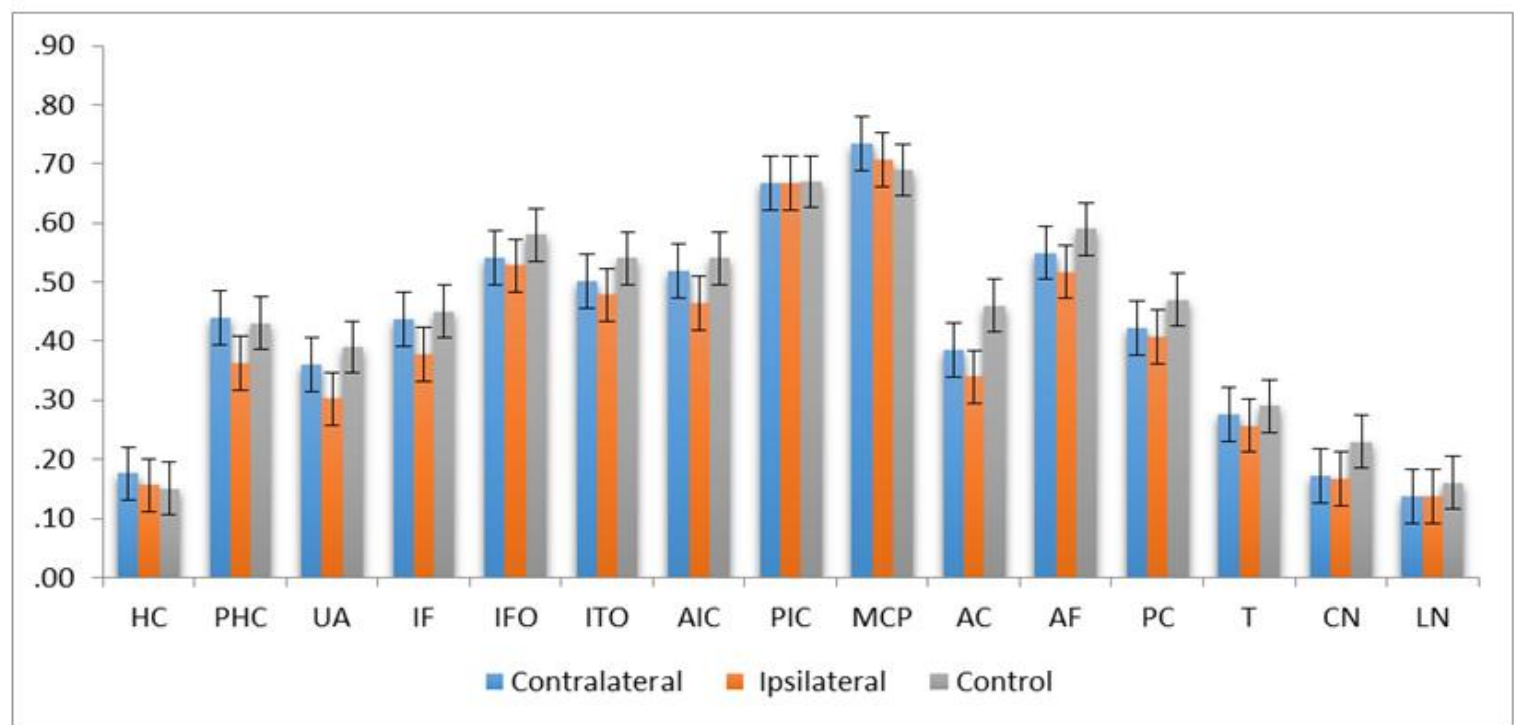

Fig .3. - Mean Fractional Anisotropy (FA) with standard deviation for each patient and mean of controls. In patients decreased Fractional Anisotropy ( FA) values noted in hippocampus ( HC), parahippocampal white matter (PHC) fornix (IF), uncinate fasciculus ( UF) anterior limb (AIC)and posterior limb(PIC) of internal capsule, superior longitudinal (IFO) and inferior Temporo-occipital (ITO) fasciculus, middle cerebellar peduncle (MCP), anterior cingulum (AC)and posterior cingulum (PC), thalamus (T), caudate nucleus (CN) and lentiform nucleus $(\mathrm{LN})$. Reduction in Fractional Anisotropy (FA) values are more in patient group as compare to controls. 

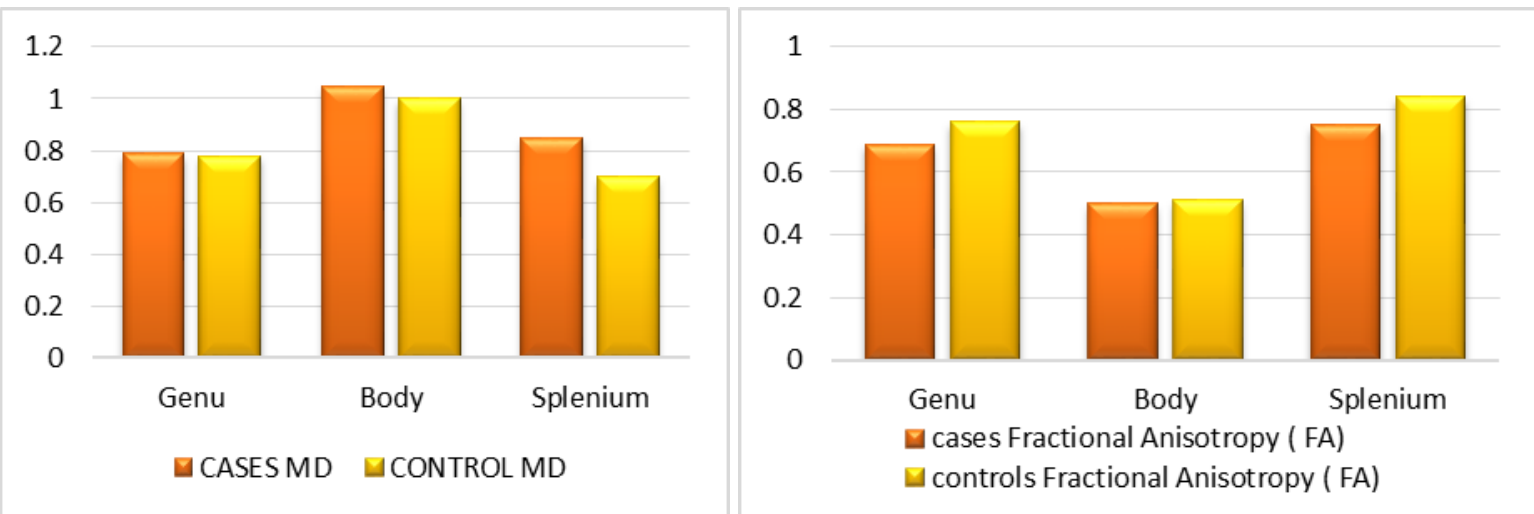

Fig .4. Bar chart of mean diffusivity (MD) and Fractional Anisotropy (FA) of corpus callosum at genu, body and splenium, comparing values of cases and controls.

\section{Discussion}

Temporal lobe epilepsy is the most common form of focal epilepsy. The hippocampus is a major source of seizure activity and microstructural alterations occur before they show up on conventional imaging ${ }^{3}$. High resolution MRI is the important modality in for the evaluation in these patients with Mesial Temporal Lobe Epilepsy (MTLE), but false negative results are also common ${ }^{30}$. During the ictal phase of seizures, there is an increase in oxygen consumption in the seizure focus, which is more than the increased blood flow. It results in relative ischemia and cytotoxic edema which is shown to result in decreased ADC. As time progresses, epilepsy results in neurogliosis, increased extracellular space and increased interictal $\mathrm{ADC}^{3}$.

a.
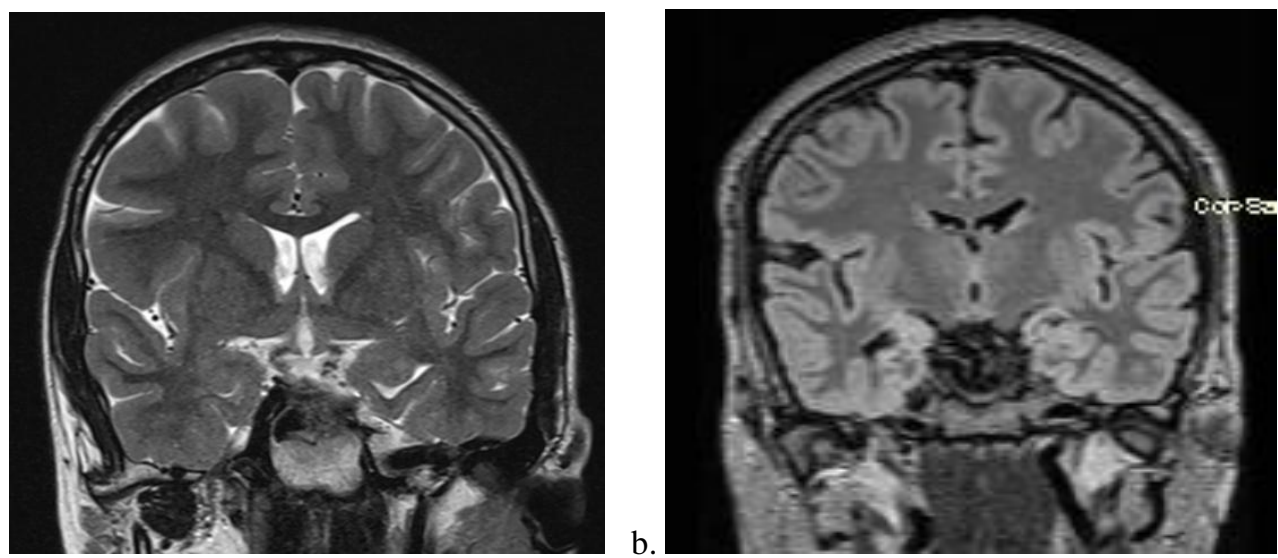

Figure 5. a. T2 coronal image showing hyperintensity with small left hippocampus in patient with left temporal lobe epilepsy suggestive of left hippocampal sclerosis, b. 3D FLAIR coronal image showing hyperintensity with small right hippocampus in patient with left temporal lobe epilepsy suggestive of left hippocampal sclerosis .

Anisotropic diffusion is also reduced in the white matter tracts through which the epileptiform discharges travel, reducing the $\mathrm{FA}^{3}$.

Diffusion tensor imaging indices are sensitive to identify these abnormalities. We have used the FA and ADC values of the hippocampi and various other grey and white matter tracts to study the hippocampal and extra hippocampal alterations.

\subsection{Hippocampal diffusivity and anisotropy:}

The hippocampal indices of 21 patients with ipsilateral hippocampal sclerosis were compared with contralateral hippocampus and the 10 age matched controls. In patients group ipsilateral hippocampus had statistically significant reduced FA and increased ADC values as compare to contralateral hippocampus. The results of our ipsilateral hippocampus is in line with the results of Thivard et $\mathrm{al}^{4}$ in 2005 and Assaf et $\mathrm{al}^{3}$ in 2003. Thivard et $\mathrm{al}^{4}$ found increased mean diffusivity (ADC) and reduced Fractional Anisotropy (FA) in the ipsilateral hippocampus and temporal lobe structures. Decreased mean diffusivity noted in contralateral hippocampus. Assaf et $\mathrm{al}^{3}$ in 2003 studied 12 patients of unilateral temporal lobe epilepsy found diffusivity measurements more sensitive than anisotropy. They found significant increase in mean diffusivity and decreased fractional anisotropy in the Hippocampal formation of ipsilateral side compared to contralateral side in patients. Comparing with the controls, the mean diffusivity remained statistically higher but the fractional anisotropy did not reach significant differences, though they were lower. 
YU Ai-hong et $\mathrm{al}^{11}$ in 2006 studied 14 patients with medial temporal lobe epilepsy and found significant decrease in mean diffusivity in ipsilateral Hippocampal formation in patient group but no significant difference was noted with Fractional Anisotropy ( FA) . Bilateral hippocampi are connected with each other through the hippocampal commissure and the anterior commissure through the fornices ${ }^{27}$. Many studies have demonstrated widespread propagation of seizure activity through the neuronal networks $5,6,7,8,9$. We have observed increased ADC and reduced FA in the contralateral hippocampus as compare to control group, the electrical activity possibly utilising these commissures.

Previous studies with contralateral hippocampus have yielded varying results as observed by Thivard et $\mathrm{al}^{4}$. The results were either normal or reduced mean diffusivity. YU Ai-hong ET al ${ }^{11}$ observed significant $^{1}$ increased in mean diffusivity in contralateral hippocampus as compared to controls but no difference in Fractional Anisotropy (FA). In our study we have observed significant increased in MD of contralateral hippocampus in all patients. This finding was consistent with previous studies showing bilateral consequences of Mesial Temporal Lobe Epilepsy (MTLE) syndrome ${ }^{17}$. All our patients had visually recognizable mesial temporal sclerosis, compared to Assaf et $\mathrm{al}^{2}$ where one third had normal conventional MR findings. These changes are marked in our study due to chronicity and visual structural changes. Though the contralateral hippocampus shows statistically significant diffusion tensor imaging indices, no such changes could be demonstrated in the fimbriae and fornices in our study. We had technical difficulties in placing the voxel exclusively within the fornix without contamination from the CSF, which might be responsible for the insignificant values. But our results concur with those of Thivard et $\mathrm{al}^{4}$ who did not find significant values in bilateral fornices.

Concha L et al ${ }^{12}$ in 2010 with 11 medically intractable TLE patients with and without hippocampal sclerosis, found positive correlation between diffusivity of fimbria - fornix and histology. DTI was acquired following an inversion pulse (TI $2200 \mathrm{~ms}$ at 1.5T) to suppress the cerebrospinal fluid signal, which likely yielded them accurate results.

\subsection{Comparison of extratemporal grey matter of patients and controls:}

We analysed the FA and ADC values of bilateral thalami, head of caudate nucleus and lentiform nucleus of all our 21 patients and compared them with 10 controls. Though there is decreased Fractional Anisotropy (FA) and increased mean diffusivity no statistical significant ADC and FA in bilateral thalamus, lentiform nuclei and the ipsilateral caudate nucleus. Our ADC results are not in accordance with Yin et al ${ }^{9}$ who got significantly altered values in the caudate nucleus and that of Chen $Q$ et al $^{5}$ who concluded that mean diffusivity is more sensitive than fractional anisotropy.

Our observation concur with Thivard et $\mathrm{al}^{4}$ with statistic parametric mapping explored the whole brain diffusion tensor imaging indices and found no statistically significant changes in the thalami . Yin et $\mathrm{al}^{5} \mathrm{had}$ found insignificant ADC values, which does concur with our results.

\subsection{Comparison of extra temporal white matter of patients and controls:}

Though the seizure onset is located within the temporal lobe, through the widespread interaction between cortical and subcortical structures, the epileptic circuitry is widened. Though structural abnormalities were limited to ipsilateral hippocampus and the temporal lobe, widespread diffusion abnormalities were seen in bilateral white matter in our study. On comparing ipsilateral and contralateral side in patient group we found significant decreased Fractional Anisotropy (FA) values in anterior cingulum, anterior limb of internal capsule, uncinate fasciculus and parahippocampal white matter. We compared other white matter tracts also though these structure shows decreased Fractional Anisotropy (FA) values on ipsilateral side but not attaining statistically significance level. (Table 1 and Fig 2\&3). Similarly we found statistical significant increased in Mean diffusivity in Inferior fronto occipital fibers and uncinate fasciculus. Other white matter tracts shows increased mean diffusivity but not attaining statistically significance level. (Table 1 and Fig 2\&3). Compared to the control group we found reduced Fractional Anisotropy ( FA) and increased Mean diffusivity in bilateral anterior limb of internal capsule, body, genu and splenium of corpus callosum, bilateral superior longitudinal fasciculus, bilateral inferior fronto -occipital fasciculus, bilateral uncinate fasciculus, ipsilateral middle cerebellar peduncle and bilateral cingulum. Our present data are in agreement with Knake et al ${ }^{13}$ who observed lower FA values in body/ trunk of corpus callosum, ipsilateral frontal and bilateral temporal lobes. They used both ROI method and whole brain analysis. The results of both methods were complementary to each other. Whole brain analysis picks up confluent changes but is insensitive to small focal changes. ROI method requires precise placement in the predetermined area. We used manually placed ROIs on preselected regions.

We have obtained statistically significant FA and ADC value in uncinate fasciculus. This shows early and preferential spread to frontal lobes through uncinate fasciculus in nearly all cases. Uncinate fasciculus is a major white matter tract connecting anterior temporal and frontal lobes. It is important in the formation and retrieval of memories and is a pathway for seizure spread to the frontal lobe ${ }^{10}$. In our study, bilateral uncinate 
fasciculus had reduced FA and increased ADC values. Diehl et al ${ }^{10}$, in 2008 analysed the DTI parameters of 28 TLE patients and correlated them with auditory and visual, immediate and delayed memory. They found significant alterations in diffusion tensor imaging indices in bilateral uncinate fasciculi correlated with memory in patients with left TLE (both medial and lateral).The involvement of uncinate fasciculus in our cases, implies impaired memory, though we did not directly test for memory.

We had statistically significant FA values in anterior cingulum, a major hippocampal pathway. This is in line with the results of Thivard et al ${ }^{4}$ who observed statistically significant differences in the cingulum. But we are not able to get significant change in posterior cingulum. The reason may be the CSF contamination at this region.

Corpus callosum is the major commissural fiber connecting the two hemispheres. Our study revealed statistically significant changes in both ADC and FA values in the body, splenium and genu of corpus callosum. Knake et $\mathrm{al}^{13}$ observed reduced FA in the genu and body of corpus callosum, Thivard et $\mathrm{al}^{4}$ found reduced FA in corpus callosum, Meng et $\mathrm{al}^{8}$ got reduced FA and increased ADC in the splenium, Yin et $\mathrm{al}^{5}$ had lower FA in genu, body and splenium with higher diffusivity in body of corpus callosum. Kim et al ${ }^{7}$ with ten patients of temporal lobe epilepsy found reduced FA values in the splenium, so our study concur with above studies.

Internal capsule is a major projection fiber. We observed statistically significant reduced FA values in anterior limb but we are not able to get significant changes in Fractional Anisotropy (FA) and Mean diffusivity posterior limb. Of the previous studies, Meng et $\mathrm{al}^{8}$ observed reduced FA and increased diffusivity values in anterior limb, posterior limb and the genu, Yin et $\mathrm{al}^{5}$ showed lower FA in the anterior and posterior limbs of internal capsule, Wang et $\mathrm{al}^{16}$ found lower FA in left posterior limb, Meng et $\mathrm{al}^{8}$ observed increased diffusivity and reduced FA in anterior and posterior limbs. We are not able to explain variable results in internal capsule in our cases.

Gross et $\mathrm{al}^{14}$, in their meta-analysis of 10 studies observed that though the cause and implications of white matter changes are unclear, they represent downstream axonal degeneration secondary to spreading seizure activity. They also observed that the changes are variable between studies in grey and white matter tract involvement and represent variations in patient selection, methodology, duration and propagation of seizure activity.

There are several limitations in our study - magnetic field strength of 3 Tesla or above could have given better results, diffusion sensitive gradient were applied in 21 direction, these can be increased in future. Slice thickness of $3 \mathrm{~mm}$ resulting in to non-isotropic image in $\mathrm{Z}$ axis, in our clinical setting because of high case load per day it is difficult to get thinner slices in $\mathrm{Z}$ axis.

\section{Conclusion}

Our study shows that Diffusion Tensor Imaging (DTI) in brain is highly sensitive method in determining microstructural changes in white matter tracts. We have seen the changes in ipsilateral and contralateral tracts which is not apparent in conventional MRI with epilepsy protocol. These changes shows the extensive white matter networks involved in the spread of epileptic waves in brain which may be the responsible for secondary generalization. Changes are more pronounced in ipsilateral hemisphere and changes in grey matter is not significant.

\section{References}

[1]. Lerner A, Mogensen M.A, Kim P.E, Shiroishi M.S, Hwang D.H ,Law M. Clinical Applications of diffusion tensor imaging ,Journal of World Neurology.2013.07.083

[2]. Assaf B.A, Mohamed F.B, Abou-Khaled K.J et al. Diffusion Tensor Imaging of Hippocampal Formation in temporal lobe epilepsy, AJNR Am J Neuroradiol 24:1857-1856, October 2003.

[3]. Thivard L, Lehericy S , Krainik A, Adam C , Dormonr D,Chiras J et al . Diffusion tensor imaging in medial temporal lobe epilepsy with hippocampal sclerosis. Journal .Neuro image.2005.06.04

[4]. Yin X .-y Qiu S .-J Liu. Z . -Y, Wang H.-z, Xiong W .-f, Li S.-s, wang Y. Extra temporal abnormalities of brain parenchyma in young adult with temporal lobe epilepsy; a diffusion tensor imaging study. Clinical radiology 69(2014) 589e 596.

[5]. Kim C.H, KOO B-B, Chung C.K , LEE J-M, Kim J.S, Lee S.K Thalamic changes in temporal lobe epilepsy with and without hippocampal sclerosis: a diffusion tensor imaging study. epilepsy research(2010)90,21,-27

[6]. Kim H, Piao Z, liu P, BingamanW, Diehl B . secondary white matter degeneration of the corpus callosum in patients with intractable temporal lobe epilepsy ; a diffusion tensor imaging study. Epilepsy research (2008)81, 136-142.

[7]. Meng L, XiagJ, Koteche R,Rose D, Zhao H, Zhao D.: White matter abnormalities in children and adolescents with temporal lobe epilepsy. Magnetic resonance imaging 28(2010)1290-1298.

[8]. Chen Q, Lui S , Li C-X, Jiang L-J, Ou-yang L, Tang H-H, et al. MRI -Negative refractive partial epilepsy : Role for diffusion tensor imaging in high field MRI. Epilepsy research (2008)80,83-89

[9]. Diehl B, Busch R,M , Duncin J.S , Piao Z ,Tkach J, Luders H.O . Abnormalities in diffusion tensor imaging of the uncinate fasiculus relate to reduced memory in temporal lobe epilepsy. Epilepsia :1-10,2008

[10]. YU Ai- hong, LI Kun-cheng, YU Chun shui et al . Diffusion tensor imaging in mesial temporal lobe epilepsy, Chinese medical journal 2006;119:1237-1241.

[11]. Concha 1, Livy D.J, Beaulieu C,Wheatley B.M, Gross D.W In vivo diffusion tensor imaging and histopathology of the fimbria fornix in temporal lobe epilepsy .The journal of Neuro Sceince , Jan 20,2010.30(3);996-1002 
[12]. Knake S, Salat D.H, Halgren E, Halko M.A, Greve D,N, Grant P.E, Changes in white matter micro structure in patients with TLE and hippocampal sclerosis, epileptic disorder $2009: 11(3) ; 244-50$.

[13]. Gross D.W, diffusion tensor imaging in temporal lobe epilepsy. Epilepsia, 52(suppl .4 ):32-34,2011

[14]. Riley J.D, Franklein D.L, Choi V, Kim C , Binder D.K, Cramer S.C et al. Diffusion tensor imaging in medial temporal lobe epilepsy with hippocampal sclerosis.Epilepsia , 51(4): 536-545,2010

[15]. Wang X -Q, Lang S - L, Hong L.U, Lim M,A,Yang -Ling M.A.O, Yang F. Changes in extra temporal integrity and cognition in tempora lobe epilepsy: a diffusion tensor imaging study.Epilepsia,51(4);536-545,2010

[16]. Spencer SS. Subtrates of localization -related epilepsies:nbiological implication of localizing findings in humans. Epilepsia 1998;39:114-123.

[17]. Coan A.C , Kubota B, Bergo F.P.G, Campos B.M , Cendes F. 3T MRI quantification of hippocampal volume and signal in mesial temporal lobe epilepsy improves detection of hippocampal sclerosis. AJNR am J Neuro radiol 35:77-83-Jan 2014 .

[18]. Duncin J.S , Imaging the brains highways - diffusion tensor imaging in epilepsy. Epilepsy current , Vol.8, No.4 2008 p.85-89

[19]. Kim C.H, Chung C K, Koo B-B, Lee J-M, Kim J.S, Lee S,K . Changes in language pathway in patients with temporal lobe epilepsy: diffusion tensor imaging analysis of the uncinate and arcuate fasciculi. World neuro surgery (3/4):509-516, March /April 2007 